\title{
Orientation-Controlled Nonradiative Energy Transfer to Colloidal Nanoplatelets: Engineering Dipole Orientation Factor
}

\author{
Onur Erdem, ${ }^{\dagger}$ Kivanc Gungor, ${ }^{\dagger, \S}$ Burak Guzelturk, $^{\dagger, \| \odot ~}$ Ibrahim Tanriover, $^{\dagger}$ Mustafa Sak, ${ }^{\dagger}$ \\ Murat Olutas, ${ }^{\dagger, \perp \odot 0}$ Didem Dede, ${ }^{\dagger, \#}$ Yusuf Kelestemur, ${ }^{\dagger, \nabla}$ and Hilmi Volkan Demir ${ }^{*}, \dagger, \ddagger \odot$ \\ ${ }^{\dagger}$ Department of Electrical and Electronics Engineering, Department of Physics, UNAM - Institute of Materials Science and \\ Nanotechnology, Bilkent University, Ankara 06800, Turkey \\ ${ }^{\ddagger}$ LUMINOUS! Center of Excellence for Semiconductor Lighting and Displays, School of Electrical and Electronic Engineering, \\ School of Physical and Mathematical Sciences, Nanyang Technological University, Nanyang Avenue, Singapore 639798, Singapore
}

Supporting Information

\begin{abstract}
We proposed and showed strongly orientationcontrolled Förster resonance energy transfer (FRET) to highly anisotropic CdSe nanoplatelets (NPLs). For this purpose, we developed a liquid-air interface self-assembly technique specific to depositing a complete monolayer of NPLs only in a single desired orientation, either fully stacked (edge-up) or fully nonstacked (facedown), with near-unity surface coverage and across large areas over $20 \mathrm{~cm}^{2}$. These NPL monolayers were employed as acceptors in an energy transfer working model system to pair with $\mathrm{CdZnS} / \mathrm{ZnS}$ core/shell quantum dots (QDs) as donors. We found the resulting energy transfer from the QDs to be significantly accelerated (by up

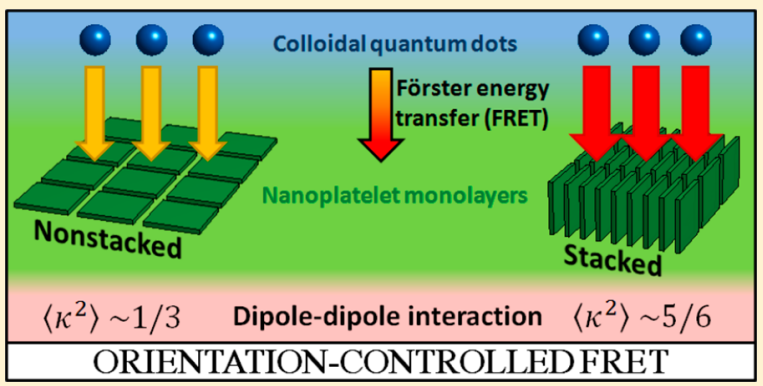
to $50 \%$ ) to the edge-up NPL monolayer compared to the face-down one. We revealed that this acceleration of FRET is accounted for by the enhancement of the dipole-dipole interaction factor between a QD-NPL pair (increased from 1/3 to 5/6) as well as the closer packing of NPLs with stacking. Also systematically studying the distance-dependence of FRET between QDs and NPL monolayers via varying their separation $(d)$ with a dielectric spacer, we found out that the FRET rate scales with $d^{-4}$ regardless of the specific NPL orientation. Our FRET model, which is based on the original Förster theory, computes the FRET efficiencies in excellent agreement with our experimental results and explains well the enhancement of FRET to NPLs with stacking. These findings indicate that the geometrical orientation of NPLs and thereby their dipole interaction strength can be exploited as an additional degree of freedom to control and tune the energy transfer rate.
\end{abstract}

KEYWORDS: semiconductor nanocrystals, nanoplatelets, liquid-air interface self-assembly, stacking, energy transfer, dipole orientation

$\mathrm{F}$ örster (or fluorescence) resonance energy transfer (FRET) is the nonradiative transfer of excitation energy from one fluorophore (donor) to another one (acceptor), which is induced by near-field interactions of oscillating dipoles. ${ }^{1,2}$ Although initial studies on FRET focused on fluorescent dyes and proteins, FRET has gained further attraction after the introduction of colloidal nanocrystals (NCs). ${ }^{3-11}$ Being an extremely distance-sensitive process, FRET involving NCs is used in a variety of applications including light-harvesting, ${ }^{5}$ optoelectronic structures, ${ }^{7}$ and biosensing. 12,13

Apart from the donor-acceptor distance, the strength of FRET depends on a number of parameters including the spectral overlap of the donor and acceptor and the radiative emission rate of the donor as well as the relative orientation of donor and acceptor dipoles. ${ }^{14}$ The last parameter, commonly denoted as $\kappa^{2}$, is especially important when either the donor or acceptor fluorophore has an anisotropic dipole state. In the case that the dipole states of both fluorophores rotate freely in three dimensions in an ensemble, $\left\langle\kappa^{2}\right\rangle$ equals $2 / 3$, which is the average value for the dipole orientation factor. ${ }^{1}$ However, for dipoles confined along one or two dimensions, or whose rotation is limited by any other means, this factor is expected to be different. ${ }^{14}$ Therefore, if the orientation of all of the anisotropic donor or acceptor species in an ensemble is controlled, we propose that the strength of FRET in semiconductor NC ensembles can be modified via tuning the average dipole-dipole interaction coefficient, $\left\langle\kappa^{2}\right\rangle$, which would serve as another degree of freedom to control FRET. However, the control of $\left\langle\kappa^{2}\right\rangle$ in NCs over macroscopic areas has remained elusive to date.

Here, to control the dipole interaction and investigate its effect on FRET, we employed colloidal semiconductor nanoplatelets (NPLs) as acceptors. As the last class of solution-processed semiconductor NCs with atomically flat

Received: February 15, 2019

Revised: May 30, 2019

Published: June 3, 2019 
surfaces and vertical-tight quantum confinement of few atomic monolayers, ${ }^{15,16}$ these free-standing NPLs make quasi twodimensional quantum wells with a very small thickness of $1-2$ $\mathrm{nm}$ and lateral dimensions ranging from several to $100 \mathrm{~s} \mathrm{~nm}$. Thus, NPLs feature intrinsically high anisotropy, because of which their transition dipole state near the band edge is mostly confined within the NPL plane. ${ }^{17}$ Furthermore, unlike other 2D materials such as epitaxially grown quantum wells and transition metal dichalcogenides, the colloidal nature of NPLs provides the ability to deposit them in an orientation of one's choice in their thin-films. ${ }^{17-19}$ This feature of NPLs, along with their unusually large extinction coefficient, ${ }^{16,18,20,21}$ makes them attractive materials as FRET acceptors with the capability of dipole interaction control.

NPLs in an ensemble might adopt one of two orientations. The former is regarded as nonstacked, or face-down, ${ }^{17,22}$ where NPLs lie flat on a substrate with their lateral surfaces being parallel to it. In the latter, i.e., stacked or edge-up orientation, ${ }^{17,23}$ NPLs stand vertically on their peripheral surfaces, face each other instead of facing down, and can form long one-dimensional NPL chains. Stacking of NPLs has been investigated on several previous reports of our group and others on various aspects. It was previously shown that stacking modifies the excitonic dynamics in NPLs through ultrafast intrastack energy transfer. ${ }^{19,24,25}$ Polarized emission out of NPL stacks has been reported on both solid ${ }^{23}$ and flexible $^{26}$ films. Stacking in NPLs was also utilized to observe their orientation-dependent emission patterns. ${ }^{17}$ These previous reports confirm that optical properties of NPLs are strongly modified with their orientation. However, with conventional methods of sample preparation such as dropcasting or spin-coating, observing NPL ensembles with mixed orientation is quite possible. ${ }^{18,21,27,28}$ To address this problem, liquid-air interface self-assembly has been employed, which enabled orientation control of platelet-shaped NCs. ${ }^{17,29}$ With this technique, nanoplates in a single orientation could be deposited in film, either as multilayers ${ }^{29}$ or as small domains with relatively low coverage on the surface. ${ }^{17}$ However, to the best of our knowledge, a uniform deposition of NPLs as a single monolayer in one particular orientation over large areas has not yet been achieved, which limits the effective utilization of anisotropy in these NPLs.

In this work, we developed a method of NPL self-assembly at a liquid-air interface, through which we successfully deposited CdSe NPLs as a single monolayer in the desired orientation over a solid substrate. The resulting self-assembled NPLs are either fully nonstacked or fully stacked. Furthermore, their surface coverage is close to unity in both cases, with domain sizes that can be as large as the area of the substrate. In our working model system, we used blue-emitting $\mathrm{CdZnS} / \mathrm{ZnS}$ core/shell QDs as the donor species on top of these NPLs and studied the strength and distance dependence of FRET between them. The measured rate of FRET from QDs to NPLs is significantly faster when using the stacked NPL monolayer compared to the nonstacked one. We found in the case of stacking that, along with the increased packing density, the enhanced dipole-dipole interaction of the stacked NPLs with the QDs accelerates FRET substantially.

We also systematically studied the distance $(d)$ dependence of FRET to the NPLs by placing $\mathrm{Al}_{2} \mathrm{O}_{3}$ as a separating layer in between the QDs and NPL monolayers and tuning its thickness, and observed that the FRET rate exhibits a $d^{-4}$ dependence, as would be expected for a plane of acceptors. ${ }^{9}$
To calculate the FRET rates in the cases of using the nonstacked vs stacked NPL acceptors, we utilized Förster's original theory of nonradiative energy transfer. In doing so, we have taken into account the uniform density of exciton states across the NPL as well as the dipole-dipole interaction strength being a function of both the position and orientation of the acceptor dipole. Our computations in both cases show excellent agreement with the FRET rates extracted from timecorrelated single photon counting experiments. This work demonstrates for the first time that the orientation of these anisotropic NPLs can be controlled over large areas and used for tuning the strength of the energy transfer by controlling the orientation of the acceptor dipole.

\section{RESULTS AND DISCUSSION}

CdSe NPLs having 4 monolayer (ML) atomic thickness and monodisperse $\mathrm{CdZnS} / \mathrm{ZnS}$ QDs were synthesized using previously reported recipes with slight modifications (see Methods). ${ }^{30,31}$ Transmission electron microscopy (TEM) image of the NPLs is depicted in Figure 1. The NPLs have

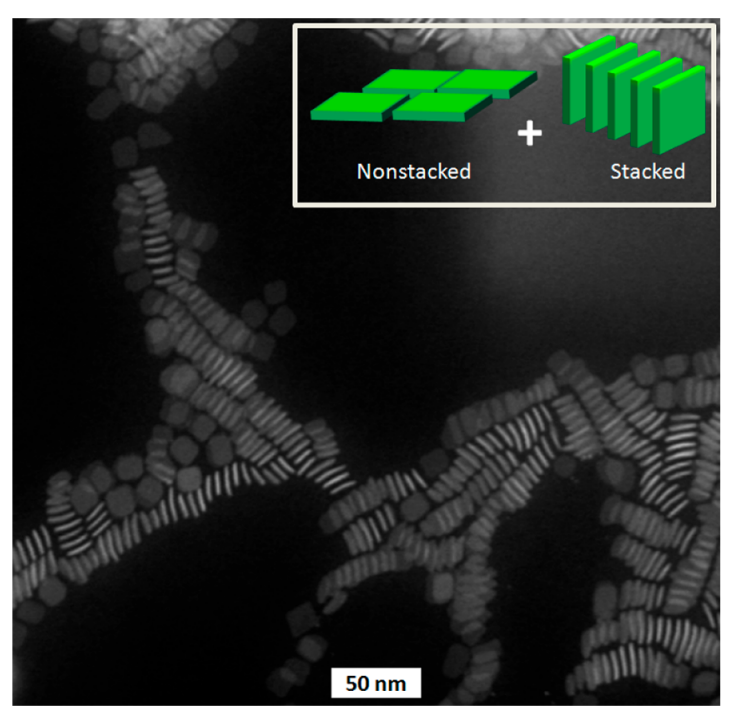

Figure 1. High-angle annular dark-field scanning transmission electron microscopy (HAADF-STEM) image of $4 \mathrm{ML}$ CdSe NPLs. Inset schematically shows the orientation of nonstacked (face-down) and stacked (edge-up) NPLs in three dimensions with the imaged facets highlighted in light green.

square-like surfaces with a side length of $14.4 \pm 2.0 \mathrm{~nm}$ as measured from the TEM images. A mixture of nonstacked and stacked NPLs with different orientations with respect to the TEM grid is visible in the image, which is typically found in NPL solid films.

For the construction of a NPL monolayer only of a single desired orientation, we developed a liquid-air interface selfassembly technique that enabled us to deposit a large-area CdSe NPL monolayer with near-unity surface coverage. Previously, liquid-air interface self-assembly was used to deposit colloidal metal nanoparticles ${ }^{32,33}$ and semiconductor QDs. ${ }^{34}$ Also, Diroll et al. demonstrated self-assembly of nanorod (NR) superlattices by using different subphases to control NR orientation. ${ }^{35}$ The interaction between the subphase and the NC and its solvent was also utilized by Paik et al. for $\mathrm{GdF}_{3}$ nanoplates to create multiple layers of "lamellar" (stacked) or "columnar" (nonstacked) assemblies of 
these nanoplates with full surface coverage over large area. ${ }^{29}$ More recently, Gao et al. studied the emission patterns of selfassembled CdSe NPL films created via liquid-air interface selfassembly. ${ }^{17}$ In this previous study, although the NPLs exhibited a uniform orientation in film with domains as large as several tens of micrometers, these domains were separated with gaps having no NPL coverage. In the present study of ours, different than previous reports, the orientation-controlled NPLs are deposited both as a single monolayer only and with full surface coverage over areas that can be as large as tens of square centimeters.

Our self-assembly procedure is illustrated in Figure $2 \mathrm{a}$. Substrates (silicon wafers containing $25 \mathrm{~nm}$ thick $\mathrm{Al}_{2} \mathrm{O}_{3}$ films

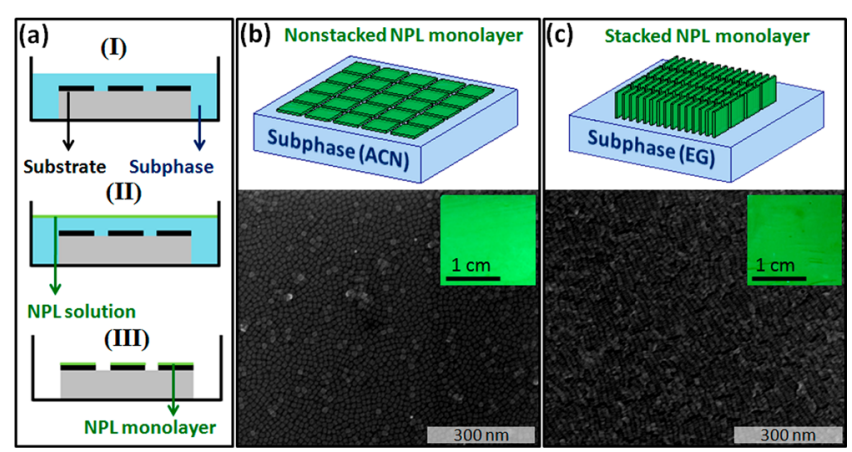

Figure 2. (a) Liquid-air interface self-assembly procedure: (I) Blank substrates are placed inside the subphase. (II) NPL solution is poured onto the subphase and is then allowed to dry. (III) The subphase is subsequently drained after the evaporation of the NPL solution. Finally, the residual subphase on the substrates is evaporated. NPL orientation on the liquid-air interface depends on the subphase. Schematic representation of NPL monolayers (b) on acetonitrile $(\mathrm{ACN})$ and (c) on ethylene glycol (EG), along with SEM images of these films after being transferred to a solid substrate. The scale bars of the SEM images are $300 \mathrm{~nm}$. ACN results in nonstacked selfassembly, whereas EG results in stack formation. Insets in the SEM images show real-color fluorescence images of the resulting monolayer assemblies on wafers of thermal oxide with scale bars of $1 \mathrm{~cm}$.

predeposited on top) are initially placed inside a subphase contained in a Teflon well. The subphase is denser than the solvent of the NPLs (hexane) and has high enough polarity to ensure its immiscibility with the NPL solution. In our approach, exploiting different surface tensions and polarities of our subphases to dictate the NPL orientation on the interface, ${ }^{29,35}$ we identified acetonitrile (ACN) as the subphase that allows to obtain a nonstacked self-assembled monolayer and ethylene glycol (EG) that yields a stacked one. Once dropped onto the subphase, the NPL solution spreads around the surface of the liquid interface. The NPL solution is then let dry, after which the NPLs on the interface form a uniform membrane. To transfer these NPLs onto substrates, the subphase is slowly drained through a needle and the residual subphase between the substrates and the NPL membrane is evaporated.

Scanning electron microscopy (SEM) images of the resulting self-assembly films are displayed in Figure $2 b$ and $c$. It can be seen that the self-assembled NPLs deposited using ACN are in a fully nonstacked orientation. Furthermore, apart from minor irregularities, the deposited NPLs form a full monolayer and are as closely packed as possible in nonstacked configuration with rare small gaps between them in the film (see Figure S2). The stacked NPLs were also deposited uniformly onto the film as a monolayer with no apparent aggregate or multilayer formation (see Figure S3). The stacks appear to be partially aligned within grains of a few square micrometers (see Figure S3a). This alignment can be further improved in principle such that all of the stacked chains in the film are aligned in the same direction. This might result in a highly polarized emitting monolayer. ${ }^{26}$ The full alignment of the chains throughout the whole substrate, however, is out of the scope of this current study and will be investigated in a future work.

By controlling the amount of NPL solution to be dropped, we are able to create domains with controlled NPL orientation over areas of at least a few square millimeters. Although this domain size is sufficient for ensemble measurements, it can be extended up to tens of square centimeters with the assistance of a surfactant (silicone oil) $)^{33}$ to further compress the monolayer NPL membrane on the liquid-air interface and reduce the crack formation during the transfer process as well as the voids. As an exemplary demonstration, 2 in. wafers of silicon oxide thermally grown on $\mathrm{Si}$, which have an area of $\sim 20$ $\mathrm{cm}^{2}$, were deposited with a monolayer of nonstacked or stacked NPLs (see Figure 2b, c insets, also Figure S4). To achieve this, we carefully controlled the concentration and the amount of NPL solution to be dropped onto the subphase such that when fully spread over the subphase, the NPLs cover virtually the whole surface of the liquid-air interface. For that, the concentration of NPL solution was estimated using the work of Yeltik et al. ${ }^{20}$

To develop a deeper understanding on how the orientation of these NPLs affects their excitonic properties, we employed the self-assembled NPLs as acceptors in an energy transfer model system. Specifically, we hypothesize that the rate of FRET from isotropic QDs to anisotropic NPLs should be modified because of the changing dipole-dipole coupling strength between a QD-NPL pair for different NPL orientations. $\mathrm{CdZnS} / \mathrm{ZnS}$ core/graded shell blue-emitting QDs dispersed in toluene were spin-coated directly onto the self-assembled NPL films. SEM images of the resulting QD films are shown on nonstacked NPLs in Figure $3 a$ and on stacked NPLs in Figure $3 \mathrm{~b}$. QDs are seen to have a submonolayer coverage on top of the NPLs, which ensures that all of the deposited QDs are equally distant to the NPL plane, and hence are coupled to the NPL film to the same extent (otherwise with a QD film thicker than one monolayer, some of the QDs would remain slightly further away to the NPLs, and FRET from those QDs would be weaker than from the QDs directly over the NPL monolayer). Since there is a significant spectral overlap between the PL emission of QDs and the absorption of the NPLs (Figure 3c), energy transfer from QDs to NPLs is expected to take place. To calculate the rate of the energy transfer, we collected the PL decays of QDs when they are deposited on top of nonstacked NPLs and stacked NPLs and compared these two decays with the decay of only-QD film (with no acceptor). Here, the decay rate of donor QDs in the presence of acceptors is modified according to

$$
\frac{1}{\tau_{\mathrm{DA}}}=\frac{1}{\tau_{\mathrm{D}}}+k_{\mathrm{T}}
$$

where $\tau_{\mathrm{D}}\left(\tau_{\mathrm{DA}}\right)$ is the PL lifetime of donor in the absence (presence) of the acceptors and $k_{\mathrm{T}}$ is the rate of energy transfer from the donor to the acceptor. 
(a)

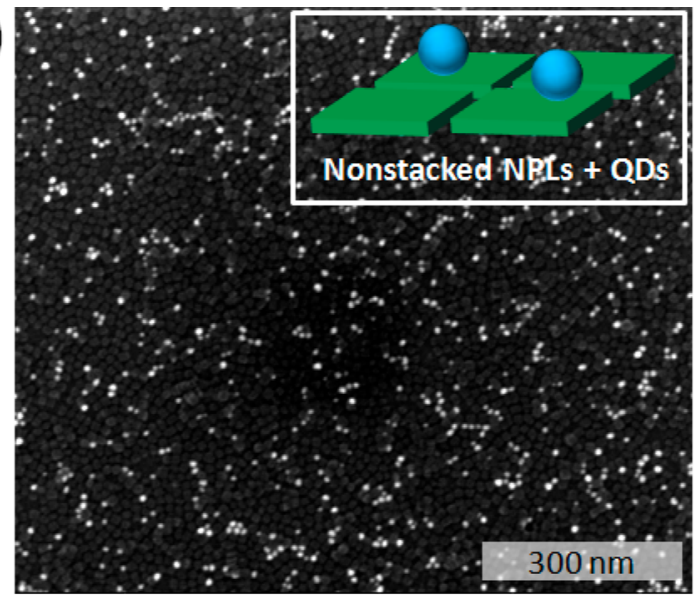

(c)

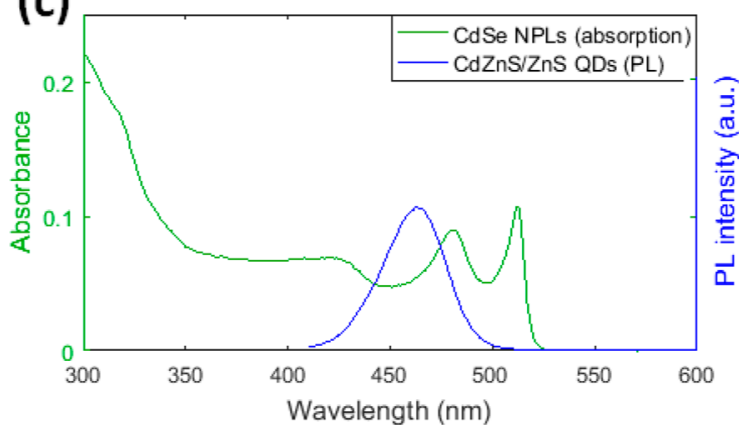

(e)

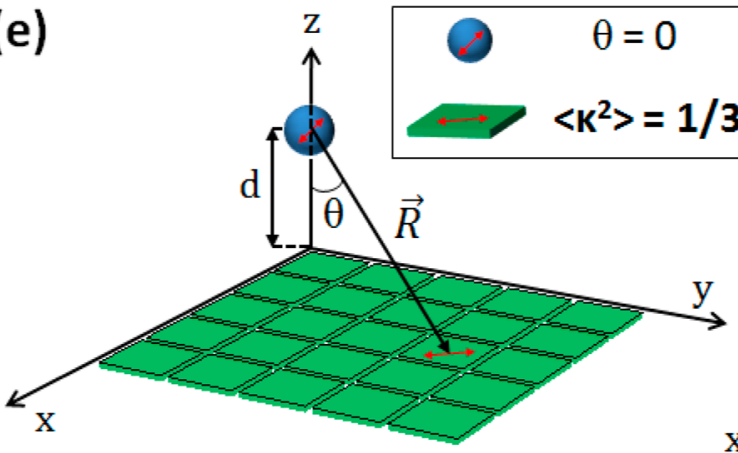

(b)

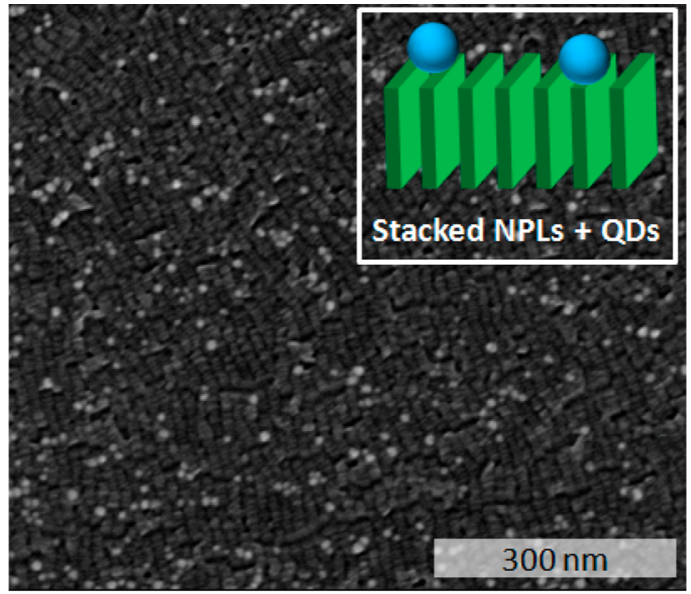

(d)

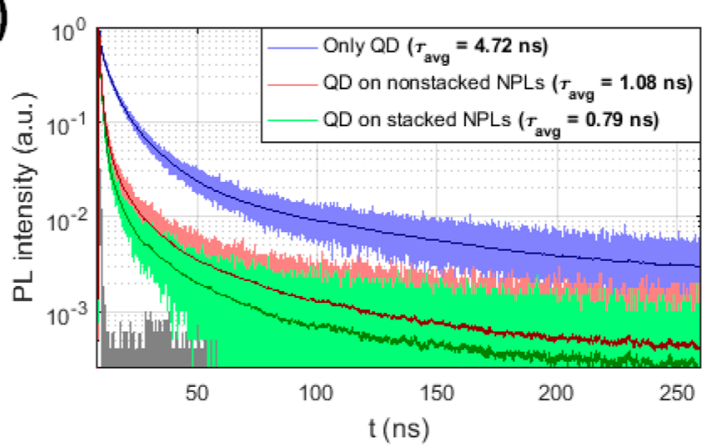

(f)

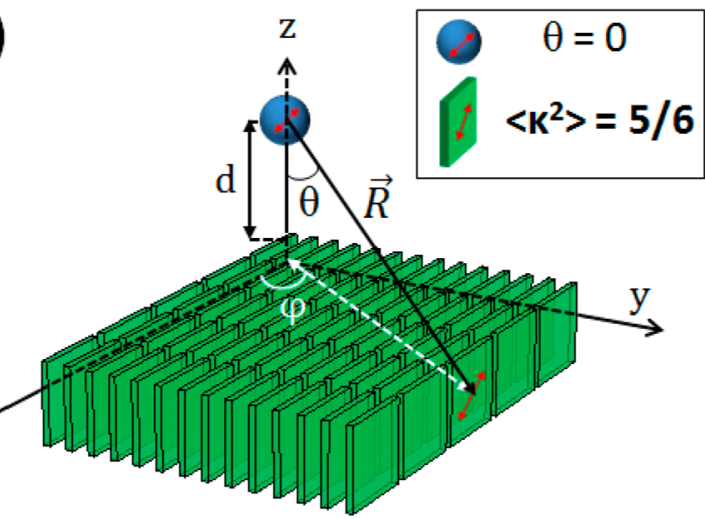

$$
\left\langle\kappa^{2}\right\rangle=\frac{2+3 \sin ^{2} \theta}{6}
$$

$$
\left\langle\kappa^{2}\right\rangle=\frac{5-3 \sin ^{2} \theta \sin ^{2} \varphi}{6}
$$

Figure 3. SEM images of CdZnS/ZnS QDs on (a) the nonstacked NPLs and (b) the stacked NPLs. (c) Absorbance spectrum of NPLs (green) and PL spectrum of QDs (blue). (d) PL decays of the QDs in the absence of NPLs (blue), on top of the nonstacked NPLs (red), and on top of the stacked NPLs (green), along with their multiexponential fits convolved with the instrument response function (gray). Average dipole-dipole interaction coefficient of a QD and an NPL monolayer when the NPLs are (e) nonstacked and (f) stacked, as a function of the dipole's position. Insets show the value of $\left\langle\kappa^{2}\right\rangle$ for a pair of the QD and the nearest NPL to it.

The PL decays of the QDs alone, the QDs on top of the nonstacked NPLs and the QDs on top of the stacked NPLs are plotted in Figure 3d. The amplitude-averaged lifetime of only QDs is $4.72 \pm 0.36 \mathrm{~ns}$, whereas in the presence of the nonstacked and stacked acceptors, the average QD lifetime reduces to $1.08 \pm 0.09$ and $0.79 \pm 0.07 \mathrm{~ns}$, respectively. Using eq 1 , these lifetimes yield FRET at the rates of $0.71 \mathrm{~ns}^{-1}$ to the nonstacked NPLs and $1.05 \mathrm{~ns}^{-1}$ to the stacked NPLs. Hence, the rate of FRET to the stacked NPL monolayer is $\sim 50 \%$ faster than to the nonstacked one. Since our domain sizes are much larger than the spot size of the excitation laser $(\sim 100 \mu \mathrm{m})$, we can assert that all the excited QDs are coupled to the nearby NPLs and hence undergo FRET, and that there is no contribution of uncoupled QDs to the PL decay in either case.

To account for the difference in the rate of FRET to the stacked and nonstacked NPL monolayers, we make use of Förster's theory of nonradiative energy transfer. ${ }^{1}$ It is also possible to account for the observed difference in the emission kinetics and energy transfer rates through Purcell effect for emitters near planarly layered media, as was previously reported; ${ }^{36,37}$ here we limit our discussion to Förster model, which allows to explain anisotropic dipole-dipole interactions 
(a)

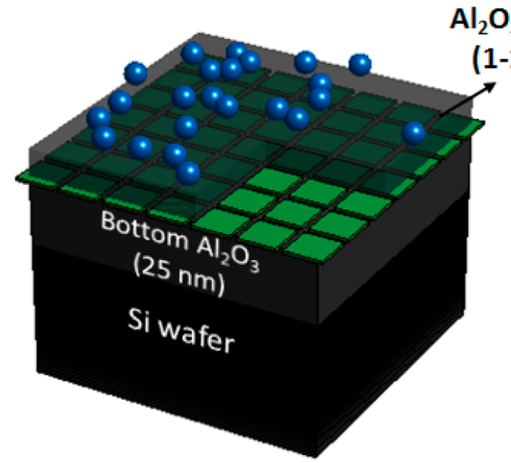

(c)

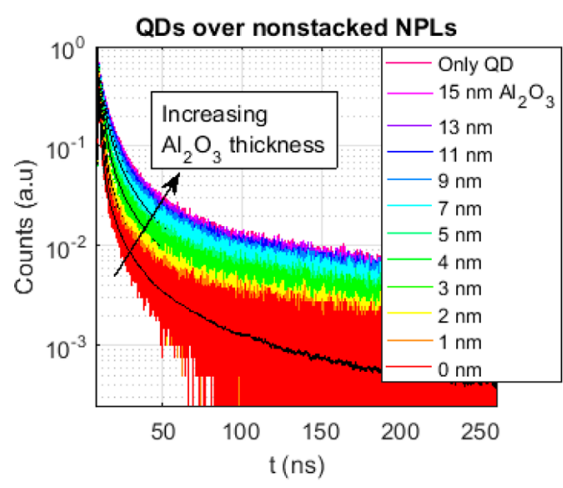

(b) $\mathrm{I}_{2} \mathrm{O}_{3}$ spacer (1-15 nm)

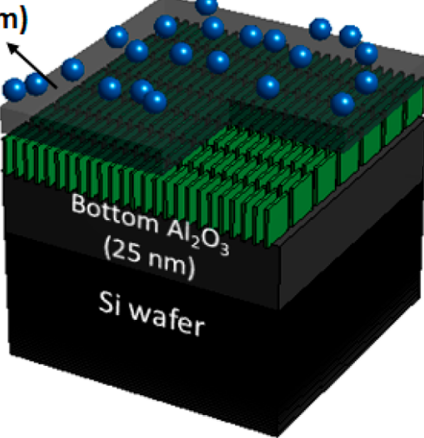

(d)

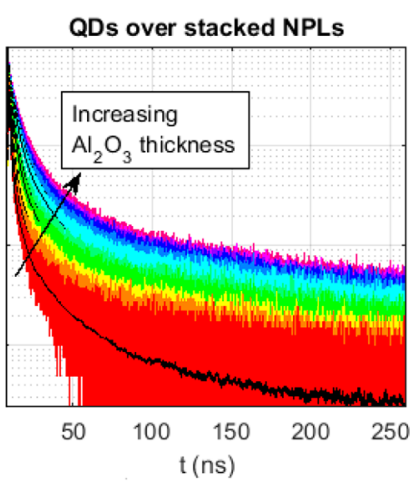

(e)

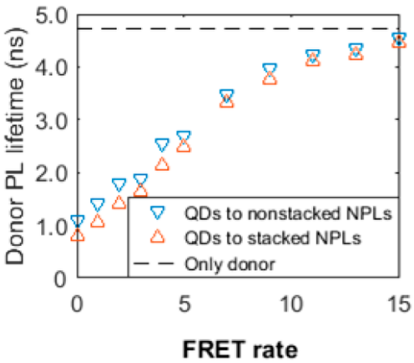

(f)

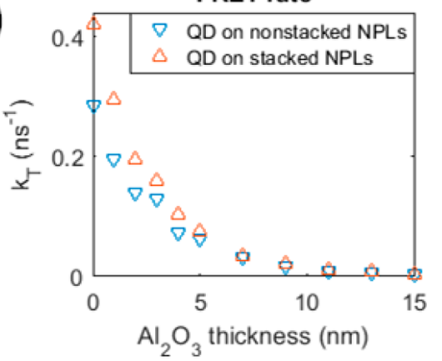

(g)

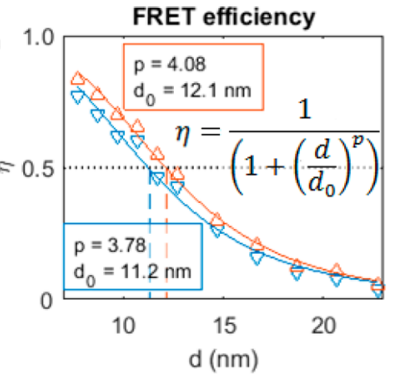

Figure 4. Film structure for distance-dependent FRET from the QDs to (a) the nonstacked NPLs and (b) the stacked NPLs. The spacer thickness is varied from 1 to $15 \mathrm{~nm}$. PL decays of QDs over (c) the nonstacked NPLs and (d) the stacked NPLs for tuned spacer thicknesses. The black curves are multiexponential fits to PL decays convolved with the instrument response function. (e) The resulting PL lifetimes of QDs and (f) extracted rates of FRET to the nonstacked (blue down triangles) and the stacked NPLs (orange up triangles). (g) FRET efficiencies as a function of the donor-acceptor distance along with their numerical fits to the FRET efficiency formula in the inset (eq 4 ). The data captures the $d^{-4}$ behavior in general for both the nonstacked and stacked NPLs. The horizontal dashed lines indicate the Förster distances for FRET to the nonstacked (blue) and stacked (orange) NPLs.

present in our case. Accordingly, the rate of energy transfer from a donor to an acceptor is proportional to the average dipole-dipole interaction coefficient $\left\langle\kappa^{2}\right\rangle$ for this pair of the donor and acceptor. When both the donor and acceptor dipoles are isotropic, $\left\langle\kappa^{2}\right\rangle$ is taken to be $2 / 3 .^{1}$ In our case, however, the acceptor dipole is mostly confined within the NPL plane, which requires $\left\langle\kappa^{2}\right\rangle$ to be evaluated accordingly. ${ }^{17}$ For an in-plane dipole of a face-down NPL, as depicted in Figure $3 \mathrm{e}$, the average of the QD-NPL dipole interaction coefficient is calculated as (see the Supporting Information),

$$
\left\langle\kappa^{2}\right\rangle=\frac{2+3 \sin ^{2} \theta}{6}
$$

where $\theta$ is the angle between the surface normal of the NPL plane and the position vector $\vec{R}$ extending from the donor dipole to the acceptor dipole (see Figure 3e). Among the NPLs on the acceptor plane, FRET will be the strongest to the NPL that is the nearest to the QD, for which $\theta$ is close to zero. Hence, $\left\langle\kappa^{2}\right\rangle$ is approximately $1 / 3$ for a $Q D$ and the closest nonstacked NPL to it. On the other hand, for the stacked monolayer of NPLs (see the Supporting Information),

$$
\left\langle\kappa^{2}\right\rangle=\frac{5-3 \sin ^{2} \theta \sin ^{2} \phi}{6}
$$

where $\phi$ is the angle between the $x$-axis and the projection of $\vec{R}$ onto the $x y$-plane (see Figure $3 \mathrm{f}$ ). Here, it is assumed that NPL stacks are aligned along one of the lateral axes, $y$. This assumption can be justified by noting the local alignment of the stacks as seen in Figures $2 c$ and $3 b$ (and Figure S3a). As a result, for a pair of the QD and the NPL closest to it in this case, $\left\langle\kappa^{2}\right\rangle=5 / 6$. Therefore, the dipole-dipole interaction between a QD and an NPL is strongly enhanced when NPLs are stacked, compared to the case in which NPLs are nonstacked.

Another difference between the stacked and nonstacked NPL monolayers is that stacking allows a much denser packing of NPLs. Taking into account the lateral area of NPLs, the length of oleate surfactants, $1.8 \mathrm{~nm},{ }^{38}$ attached to the NPL surfaces, and the center-to-center distance of $4.3 \mathrm{~nm}$ between neighboring $4 \mathrm{ML}$ CdSe NPLs in a stack, ${ }^{19}$ the surface density of NPLs has been estimated to be $3.09 \times 10^{3} \mu \mathrm{m}^{-2}$ for the nonstacked NPLs and $1.29 \times 10^{4} \mu \mathrm{m}^{-2}$ for the stacked NPLs. Therefore, there is almost a 4-fold increase in packing density with the stacking of NPLs. This means that more NPLs can be in close proximity of a QD in the case of stacking. Moreover, the dipole interaction will be stronger for these NPLs compared to the nonstacked ones, as discussed above. Combining these two factors, it can be expected for the strength of FRET to increase with stacking of NPLs. 
To develop a quantitative analysis on how FRET is affected by the NPL orientation, we investigated the strength of FRET by varying the distance between the QDs and the NPL monolayer in a systematic way. For this purpose, we added a spacer of $\mathrm{Al}_{2} \mathrm{O}_{3}$ via atomic layer deposition, whose film thickness is swept from 1 to $15 \mathrm{~nm}$, between the QDs and selfassembled NPL monolayer. The overall structure is schematically depicted in Figure 4a for the nonstacked NPLs and in Figure $4 \mathrm{~b}$ for the stacked NPLs. There is also a bottom $\mathrm{Al}_{2} \mathrm{O}_{3}$ film, which is deposited directly on $\mathrm{Si}$ substrate to avoid energy transfer from the QDs to the Si wafer. ${ }^{39-41}$ NPLs were laid down via the described self-assembly procedure. Using our setup, which is capable of depositing up to $121 \times 1 \mathrm{~cm}^{2}$ substrates simultaneously (see Figure S1), a set of NPL films (i.e., fully nonstacked or fully stacked) were deposited at once to ensure all the NPL films are prepared under the same experimental conditions. Diluted solution of QDs was spin coated onto the $\mathrm{Al}_{2} \mathrm{O}_{3}$ spacer or directly onto the NPL monolayer (no spacer case). The PL decays of QDs are plotted in Figure $4 \mathrm{c}$ and $\mathrm{d}$ for the QDs over the nonstacked and stacked NPLs, respectively. The decays of films without a spacer, displayed in Figure $3 \mathrm{~d}$, are also added here, labeled as " $0 \mathrm{~nm} \mathrm{Al} \mathrm{Al}_{2}$ " in the legend. It can be seen that as the spacer thickness decreases, the decays are progressively accelerated, indicating FRET growing increasingly stronger. The amplitude-averaged PL lifetimes as a function of the $\mathrm{Al}_{2} \mathrm{O}_{3}$ film thickness are plotted in Figure 4e. The PL lifetimes converge to that of only donor with increasing spacer thickness as expected. The corresponding rates of FRET are calculated using eq 1 and presented in Figure $4 f$. For all the spacer thicknesses, FRET is stronger in the case of stacked NPLs. Finally, Figure $4 \mathrm{~g}$ depicts the efficiency of FRET from the QDs to the nonstacked and stacked NPL monolayers. The FRET efficiency is given by

$$
\eta=\frac{1}{1+\left(\frac{d}{d_{0}}\right)^{p}}
$$

where $d_{0}$ is the Förster distance at which the efficiency is $50 \%$, and $p$ is the exponent indicating the dependence of FRET rate on the distance; it is 6 for a pair of point-like donor and acceptor and is lower for higher acceptor dimensionalities. The effective distance $d$ here is defined from the center of the $\mathrm{QD}$ to the plane of acceptors (see Figure $3 \mathrm{e}, \mathrm{f}$ ), as is commonly used in hybrid FRET systems employing QDs. ${ }^{42,43}$ The FRET efficiency was calculated from the lifetime data using $\eta=1-\tau_{\mathrm{DA}} / \tau_{\mathrm{D}}$ and fit to eq 4 to estimate the Förster distance as well as the distance-dependence of the FRET. For the nonstacked NPL monolayer, the best fit parameters are $d_{0}$ $=11.2 \mathrm{~nm}$ and $p=3.78$, whereas for the stacked NPL monolayer they are $d_{0}=12.1 \mathrm{~nm}$ and $p=4.08$. Therefore, the distance dependence in both cases is close to $d^{-4}$ as expected for FRET to the $2 \mathrm{D}$ materials ${ }^{44-46}$ or to plane of acceptors. ${ }^{9,47}$

To calculate the FRET rate from the QDs to the nonstacked or stacked NPLs, we make use of Förster's treatment of energy transfer. Accordingly, the rate of energy transfer $k_{\mathrm{T}}$ from a donor species to an acceptor one is ${ }^{14}$

$$
k_{\mathrm{T}}=\frac{\left\langle\kappa^{2}\right\rangle \mathrm{QY}}{r^{6} \tau_{\mathrm{D}}} \frac{9 \ln 10}{128 \pi^{5} N n^{4}} J
$$

where $Q Y$ is the PL quantum yield of the donor species in the absence of acceptors, $r$ is the distance between the donor and acceptor, $N$ is Avogadro's number, $n$ is the refractive index of the medium and $J$ is the spectral overlap between the emission of the donor and absorbance of the acceptor, given by

$$
J=\int_{0}^{\infty} F_{D}(\lambda) \varepsilon_{\mathrm{A}}(\lambda) \lambda^{4} \mathrm{~d} \lambda
$$

Here $F_{D}(\lambda)$ is the normalized PL intensity spectrum and $\varepsilon_{A}(\lambda)$ is the wavelength-dependent molar extinction coefficient of the NPLs. The QY of our QDs were measured to be $65 \%$ in film. $n$ $=1.72$ was used as the refractive index of the medium. For FRET systems where the size of the donor and acceptor are much smaller than their separation, $r$ can be taken as the center-to-center distance between the donor and the acceptor. However, this is not the case with our NPLs, as their size is comparable to the QD-NPL separation. Assuming the dipole is centered inside the NPL is therefore not very suitable. Instead, we use an approach that has been utilized by Shafran et al. previously for FRET from QDs to carbon nanotubes, where they proposed a stochastic model in which the probability of FRET to a particular point on a carbon nanotube is proportional to the FRET rate calculated by eq 5 and calculated the total rate of FRET to anywhere on the carbon nanotube accordingly. ${ }^{48} \mathrm{We}$ approximate the NPLs as infinitesimally thin quantum wells with lateral dimensions much larger than the exciton Bohr radius, in which case the density of states available for energy transfer would be uniformly distributed across the NPL cross-sectional area. Then, the rate of energy transfer to a single NPL is

$$
k_{\mathrm{T}, \mathrm{NPL}}=\frac{1}{A} \iint_{A} k_{\mathrm{T} \mid \vec{r}^{\prime}} \mathrm{d} A^{\prime}
$$

where $k_{\mathrm{T} r^{\prime}}$ is the FRET rate for an acceptor dipole state localized at $\vec{r}^{\prime}$ as calculated using eq 5 . The dipole-dipole distance for a particular position $\vec{r}^{\prime}$ on the NPL is $r=\left|\vec{R}_{0}+\vec{r}^{\prime}\right|$, where $R_{0}$ is the center-to-center distance between the donor and the acceptor NCs (see Figure 5a and b). Similarly, $\left\langle\kappa^{2}\right\rangle$ will depend on $\vec{r}^{\prime}$ through eq 2 for the nonstacked NPL ensemble and eq 3 for the stacked one. For a particular NPL, the integral in eq 7 is taken over the NPL cross-sectional area. The total FRET rate to the NPL monolayer is then found by adding up the FRET rates from a QD to all the NPLs in the monolayer. The FRET rates to the nonstacked and stacked NPL monolayers estimated by this approach are plotted in Figure $5 \mathrm{c}$ and $\mathrm{d}$, respectively, together with the corresponding rates calculated from the experimental data. In both the nonstacked and stacked cases, we observe an excellent agreement between the delocalized dipole approach and the experimental data.

To compare the predictions of this model with those of a conventional center-to-center distance approach, we also plotted the estimated FRET rates assuming the dipole states in the NPLs are centered in the NPLs (Figure 5c and d; black dots). It is clearly seen that the FRET rates calculated using the center-to-center distances do not agree with the experiment for small spacer thicknesses. The centered dipole approach overshoots the experimentally measured FRET rates for the nonstacked NPL monolayer and underestimates them for the stacked one progressively with decreasing spacer thickness in few nanometer range, with this discrepancy maximized in the no spacer case. It is, however, worth noting that as the donoracceptor separation increases, the estimations of both models converge to and agree well with the experimental FRET rates. This is the expected behavior since for large enough 


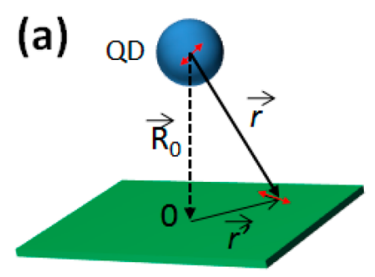

NPL (face-down)

(c)

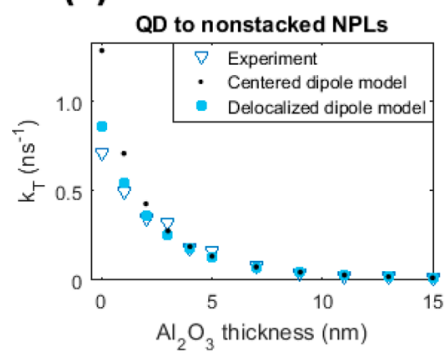

Figure 5. Variation of distance between a QD dipole and an NPL dipole for (a) nonstacked and (b) stacked NPL orientation, depending on the position $\vec{r}^{\prime}$ of the NPL dipole state. (c) Experimental rates of FRET to the nonstacked NPL monolayer (blue down triangle) compared to those estimated by two different models based on Förster's theory: center-to-center distance approach (small black dots) and delocalized dipole approach (large blue dots). (d) Experimental rates of FRET to the stacked NPL monolayer (orange up triangle) compared to the those estimated by center-tocenter distance approach (small black dots) and delocalized dipole approach (large red dots). The delocalized dipole approach shows an excellent agreement with the experimental data whereas the centered dipole approach yields increasing discrepancy as the spacer thickness is diminishing.

separations, the donor and acceptor can be approximated as point dipoles located at the donor and acceptor centers.

In conclusion, we proposed and demonstrated that controlled orientation of self-assembled NPL monolayers can be used to modify and control the strength of energy transfer via tuning of dipole-dipole interaction. We deposited NPLs in a desired orientation of either face-down or edge-up only and as a full monolayer over areas as large as $20 \mathrm{~cm}^{2}$ using a liquid-air self-assembly technique. We showed that the FRET performance of the films substantially differ in each orientation. FRET from QDs to a monolayer of edge-up NPLs is about $50 \%$ stronger than to the face-down ones, whereas the distance dependence of their FRET rates is very similar and goes by $\sim d^{-4}$ in both cases as expected for a plane of acceptors. The enhancement of FRET with stacking can be explained by the stronger dipole-dipole coupling and higher packing density. Our approach utilizing Förster theory and taking dipole state delocalization as well as different dipole-dipole interaction of QDs with nonstacked and stacked NPLs into account can successfully estimate the FRET rates from the QDs to the nonstacked and stacked NPL monolayers. Our study reveals a tool to use dipole interaction of NPLs with other NCs as an additional degree of freedom for tuning the strength of FRET and sheds further light into the orientation-dependent optical properties of anisotropic colloidal semiconductor NPLs.

\section{METHODS}

Synthesis of CdSe NPLs. CdSe NPLs having 4 monolayers (ML) of atomic thickness were synthesized using a previously reported recipe with slight modifications. ${ }^{30}$ Initially, $340 \mathrm{mg}$ of cadmium myristate and $24 \mathrm{mg}$ of selenium (Se) in $30 \mathrm{~mL}$ of ODE were degassed and stirred at $95{ }^{\circ} \mathrm{C}$ under vacuum for an hour. Afterward, the temperature was set to $240{ }^{\circ} \mathrm{C}$ and the vacuum was broken at 100 ${ }^{\circ} \mathrm{C}$ using argon gas. As the temperature reached about $195{ }^{\circ} \mathrm{C}$, the color of solution became bright yellowish and $120 \mathrm{mg}$ of cadmium acetate dihydrate $\left(\mathrm{Cd}(\mathrm{OAc})_{2} \cdot 2 \mathrm{H}_{2} \mathrm{O}\right)$ was introduced swiftly into the reaction solution. After $10 \mathrm{~min}$ of growth of NPLs at $240{ }^{\circ} \mathrm{C}$, the reaction was stopped with the injection of $1 \mathrm{~mL}$ of oleic acid (OA) and the temperature of the solution was cooled down to room temperature using a water bath. Subsequently, the resulting solution was centrifuged for $5 \mathrm{~min}$ at $6000 \mathrm{rpm}$ and the supernatant was removed into another centrifuge tube. After addition of ethanol into the supernatant solution until it became turbid, the solution was centrifuged again at $6000 \mathrm{rpm}$ for $10 \mathrm{~min}$, and finally the precipitate of 4 ML CdSe NPLs was dispersed in hexane.

Synthesis of CdZnS/ZnS Core-Graded Shell QDs. The synthesis of blue-emitting QDs was performed according to the procedure in the literature with slight modifications. ${ }^{31} 1 \mathrm{mmol} \mathrm{CdO}$, $10 \mathrm{mmol} \mathrm{Zn}$ acetate and $7 \mathrm{~mL}$ of $\mathrm{OA}$ were loaded into a $100 \mathrm{~mL}$ three-neck flask and stirred around $1 \mathrm{~h}$ at $115{ }^{\circ} \mathrm{C}$ under vacuum condition. Then, $15 \mathrm{~mL}$ of ODE was added to the mixture and further degassed for about half an hour. Under Ar atmosphere, the reaction mixture was heated up to $300{ }^{\circ} \mathrm{C}$. At this temperature, the first sulfur solution was quickly injected $(1.6 \mathrm{mmol} \mathrm{S}$ dissolved in $2.4 \mathrm{~mL}$ ODE at $100{ }^{\circ} \mathrm{C}$ ). After $12 \mathrm{~min}, 5 \mathrm{~mL}$ of $0.8 \mathrm{M}$ sulfur precursor $(4.8 \mathrm{mmol} \mathrm{S}$ dissolved in $6 \mathrm{~mL} \mathrm{OA}$ at $140{ }^{\circ} \mathrm{C}$ ) was injected at a rate of $0.5 \mathrm{~mL} / \mathrm{h}$. One hour later, remaining $1 \mathrm{~mL}$ of sulfur solution was injected with the same rate for the further growth of $\mathrm{ZnS}$ shell growth. When the reaction completed, the mixture cooled down to room temperature in water bath. For the purification step, QDs were cleaned a few times with isopropanol/ethanol mixture at $10,000 \mathrm{rpm}$ and finally redispersed in toluene.

PL Decay Measurements. A FluoTime 200 time-resolved spectrometer with a temporal resolution of 4 ps was used to measure the PL decays of only-QD and QD-NPL hybrid films at the QD emission peak of $\sim 460 \mathrm{~nm}$. The excitation laser has a wavelength of $375 \mathrm{~nm}$, a pulse width of $\sim 200 \mathrm{ps}$, a pulse repetition rate of $2.5 \mathrm{MHz}$, and a spot size of $\sim 100 \mu \mathrm{m}$. The PL decays were fit to multiexponential decays $\sum_{i} A_{i} \mathrm{e}^{-t / \tau_{i}}$ convolved with the instrument response function. The amplitude-averaged lifetimes were calculated as $\tau_{\text {avg }}=\sum_{i}\left(A_{i} \tau_{i}\right) / \sum_{i} A_{i}$.

\section{ASSOCIATED CONTENT}

\section{Supporting Information}

The Supporting Information is available free of charge on the ACS Publications website at DOI: 10.1021/acs.nanolett.9b00681.

Photographs of the self-assembly experimental setup and thermal oxide wafers deposited with NPLs under UVlight; additional SEM images of nonstacked and stacked NPL monolayer films; derivation of the positiondependent average dipole-dipole interaction coefficient for QDs and nonstacked/stacked NPLs; PL decay fitting parameters (PDF)

\section{AUTHOR INFORMATION}

\section{Corresponding Author}

*E-mail: volkan@stanfordalumni.org.

ORCID $\odot$

Burak Guzelturk: 0000-0003-1977-6485

Murat Olutas: 0000-0002-6250-6977 
Hilmi Volkan Demir: 0000-0003-1793-112X

\section{Present Addresses}

${ }^{\S}$ K.G.: Chemistry Division, Los Alamos National Laboratory, Los Alamos, NM 87545, USA.

"B.G.: Department of Materials Science and Engineering, Stanford University, Stanford, CA 94305, USA.

${ }^{\perp}$ M.O.: Department of Physics, Abant Izzet Baysal University, Bolu 14030, Turkey.

${ }^{\#}$ D.D.: Materials Science and Engineering Department, École Polytechnique Fédérale de Lausanne, 1015, Switzerland.

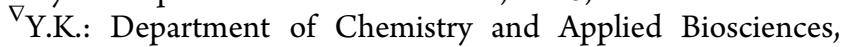
ETH Zürich, Zürich, CH 8093, Switzerland.

\section{Notes}

The authors declare no competing financial interest.

\section{ACKNOWLEDGMENTS}

The authors acknowledge the financial support from the Singapore National Research Foundation under the program of NRF-NRFI2016-08 and in part from TUBITAK 115E679. H.V.D. also gratefully acknowledges support from TUBA. O.E., K.G., I.T., M.S., and Y.K. acknowledge TUBITAK for their financial support through BIDEB program.

\section{REFERENCES}

(1) Förster, T. Zwischenmolekulare Energiewanderung Und Fluoreszenz. Ann. Phys. 1948, 437, 55-75.

(2) Kuhn, H. Classical Aspects of Energy Transfer in Molecular Systems. J. Chem. Phys. 1970, 53, 101-108.

(3) Kagan, C. R.; Murray, C. B.; Nirmal, M.; Bawendi, M. G. Electronic Energy Transfer in CdSe Quantum Dot Solids. Phys. Rev. Lett. 1996, 76, 1517-1520.

(4) Tang, Z.; Ozturk, B.; Wang, Y.; Kotov, N. A. Simple Preparation Strategy and One-Dimensional Energy Transfer in CdTe Nanoparticle Chains. J. Phys. Chem. B 2004, 108, 6927-6931.

(5) Franzl, T.; Klar, T. A.; Schietinger, S.; Rogach, A. L.; Feldmann, J. Exciton Recycling in Graded Gap Nanocrystal Structures. Nano Lett. 2004, 4, 1599-1603.

(6) Achermann, M.; Petruska, M. A.; Kos, S.; Smith, D. L.; Koleske, D. D.; Klimov, V. I. Energy-Transfer Pumping of Semiconductor Nanocrystals Using an Epitaxial Quantum Well. Nature 2004, 429, 642-646.

(7) Becker, K.; Lupton, J. M.; Müller, J.; Rogach, A. L.; Talapin, D. V.; Weller, H.; Feldmann, J. Electrical Control of Förster Energy Transfer. Nat. Mater. 2006, 5, 777-781.

(8) Clapp, A. R.; Medintz, I. L.; Mauro, J. M.; Fisher, B. R.; Bawendi, M. G.; Mattoussi, H. Fluorescence Resonance Energy Transfer Between Quantum Dot Donors and Dye-Labeled Protein Acceptors. J. Am. Chem. Soc. 2004, 126, 301-310.

(9) Rogach, A. L.; Klar, T. a.; Lupton, J. M.; Meijerink, A.; Feldmann, J. Energy Transfer with Semiconductor Nanocrystals. J. Mater. Chem. 2009, 19, 1208.

(10) Guzelturk, B.; Demir, H. V. Near-Field Energy Transfer Using Nanoemitters For Optoelectronics. Adv. Funct. Mater. 2016, 26, $8158-8177$.

(11) Alivisatos, A. P.; Gu, W.; Larabell, C. Quantum Dots as Cellular Probes. Annu. Rev. Biomed. Eng. 2005, 7, 55-76.

(12) Willard, D. M.; Carillo, L. L.; Jung, J.; Van Orden, A. CdSe-ZnS Quantum Dots as Resonance Energy Transfer Donors in a Model Protein-Protein Binding Assay. Nano Lett. 2001, 1, 469-474.

(13) Medintz, I. L.; Clapp, A. R.; Mattoussi, H.; Goldman, E. R.; Fisher, B.; Mauro, J. M. Self-Assembled Nanoscale Biosensors Based on Quantum Dot FRET Donors. Nat. Mater. 2003, 2, 630-638.

(14) Clegg, R. M. Fluorescence Resonance Energy Transfer. In Fluorescence Imaging Spectroscopy and Microscopy; Wang, X. F., Herman, B., Eds.; Wiley: New York, 1996; pp 179-252.
(15) Ithurria, S.; Dubertret, B. Quasi 2D Colloidal CdSe Platelets with Thicknesses Controlled at the Atomic Level. J. Am. Chem. Soc. 2008, 130, 16504-16505.

(16) Ithurria, S.; Tessier, M. D.; Mahler, B.; Lobo, R. P. S. M.; Dubertret, B.; Efros, A. L. Colloidal Nanoplatelets with TwoDimensional Electronic Structure. Nat. Mater. 2011, 10, 936-941.

(17) Gao, Y.; Weidman, M. C.; Tisdale, W. A. CdSe Nanoplatelet Films with Controlled Orientation of Their Transition Dipole Moment. Nano Lett. 2017, 17, 3837-3843.

(18) She, C.; Fedin, I.; Dolzhnikov, D. S.; Demortière, A.; Schaller, R. D.; Pelton, M.; Talapin, D. V. Low-Threshold Stimulated Emission Using Colloidal Quantum Wells. Nano Lett. 2014, 14, 2772-2777.

(19) Guzelturk, B.; Erdem, O.; Olutas, M.; Kelestemur, Y.; Demir, H. V. Stacking in Colloidal Nanoplatelets: Tuning Excitonic Properties. ACS Nano 2014, 8, 12524-12533.

(20) Yeltik, A.; Delikanli, S.; Olutas, M.; Kelestemur, Y.; Guzelturk, B.; Demir, H. V. Experimental Determination of the Absorption Cross-Section and Molar Extinction Coefficient of Colloidal CdSe Nanoplatelets. J. Phys. Chem. C 2015, 119, 26768-26775.

(21) Guzelturk, B.; Kelestemur, Y.; Olutas, M.; Delikanli, S.; Demir, H. V. Amplified Spontaneous Emission and Lasing in Colloidal Nanoplatelets. ACS Nano 2014, 8, 6599-6605.

(22) Antanovich, A.; Prudnikau, A.; Matsukovich, A.; Achtstein, A. W.; Artemyev, M. Self-Assembly of CdSe Nanoplatelets into Stacks of Controlled Size Induced by Ligand Exchange. J. Phys. Chem. C 2016, 120, 5764-5775.

(23) Abécassis, B.; Tessier, M. D.; Davidson, P.; Dubertret, B. SelfAssembly of CdSe Nanoplatelets into Giant Micrometer-Scale Needles Emitting Polarized Light. Nano Lett. 2014, 14, 710-715.

(24) Rowland, C. E.; Fedin, I.; Zhang, H.; Gray, S. K.; Govorov, A. O.; Talapin, D. V.; Schaller, R. D. Picosecond Energy Transfer and Multiexciton Transfer Outpaces Auger Recombination in Binary CdSe Nanoplatelet Solids. Nat. Mater. 2015, 14, 484-489.

(25) Erdem, O.; Olutas, M.; Guzelturk, B.; Kelestemur, Y.; Demir, H. V. Temperature-Dependent Emission Kinetics of Colloidal Semiconductor Nanoplatelets Strongly Modified by Stacking. J. Phys. Chem. Lett. 2016, 7, 548-554.

(26) Beaudoin, E.; Abecassis, B.; Constantin, D.; Degrouard, J.; Davidson, P. Strain-Controlled Fluorescence Polarization in a CdSe Nanoplatelet-Block Copolymer Composite. Chem. Commun. 2015, 51, 4051-4054A.

(27) Feng, F.; Nguyen, L. T.; Nasilowski, M.; Nadal, B.; Dubertret, B.; Maître, A.; Coolen, L. Probing the Fluorescence Dipoles of Single Cubic CdSe/CdS Nanoplatelets with Vertical or Horizontal Orientations. ACS Photonics 2018, 5, 1994-1999.

(28) Yang, G.; Kazes, M.; Oron, D. Chiral 2D Colloidal Semiconductor Quantum Wells. Adv. Funct. Mater. 2018, 28, 1802012.

(29) Paik, T.; Ko, D. K.; Gordon, T. R.; Doan-Nguyen, V.; Murray, C. B. Studies of Liquid Crystalline Self-Assembly of GdF3 Nanoplates by in-Plane, out-of-Plane SAXS. ACS Nano 2011, 5, 8322-8330.

(30) Tessier, M. D.; Spinicelli, P.; Dupont, D.; Patriarche, G.; Ithurria, S.; Dubertret, B. Efficient Exciton Concentrators Built from Colloidal Core/Crown CdSe/CdS Semiconductor Nanoplatelets. Nano Lett. 2014, 14, 207-213.

(31) Lee, K. H.; Lee, J. H.; Song, W. S.; Ko, H.; Lee, C.; Lee, J. H.; Yang, H. Highly Efficient, Color-Pure, Color-Stable Blue Quantum Dot Light-Emitting Devices. ACS Nano 2013, 7, 7295-7302.

(32) Narayanan, S.; Wang, J.; Lin, X. M. Dynamical Self-Assembly of Nanocrystal Superlattices during Colloidal Droplet Evaporation by in Situ Small Angle x-Ray Scattering. Phys. Rev. Lett. 2004, 93, 135503.

(33) Min, H.; Zhou, J.; Bai, X.; Li, L.; Zhang, K.; Wang, T.; Zhang, X.; Li, Y.; Jiao, Y.; Qi, X.; et al. Approach to Fabricating a Compact Gold Nanoparticle Film with the Assistance of a Surfactant. Langmuir 2017, 33, 6732-6738.

(34) Dong, A.; Chen, J.; Vora, P. M.; Kikkawa, J. M.; Murray, C. B. Binary Nanocrystal Superlattice Membranes Self-Assembled at the Liquid-Air Interface. Nature 2010, 466, 474-477. 
(35) Diroll, B. T.; Greybush, N. J.; Kagan, C. R.; Murray, C. B. Smectic Nanorod Superlattices Assembled on Liquid Subphases: Structure, Orientation, Defects, and Optical Polarization. Chem. Mater. 2015, 27, 2998-3008.

(36) Nguyen, H. M.; Seitz, O.; Peng, W.; Gartstein, Y. N.; Chabal, Y. J.; Malko, A. V. Efficient Radiative and Nonradiative Energy Transfer from Proximal CdSe/ZnS Nanocrystals into Silicon Nanomembranes. ACS Nano 2012, 6, 5574-5582.

(37) Pelton, M. Modified Spontaneous Emission in Nanophotonic Structures. Nat. Photonics 2015, 9, 427-435.

(38) Urban, J. J.; Talapin, D. V.; Shevchenko, E. V.; Murray, C. B. Self-Assembly of $\mathrm{PbTe}$ Quantum Dots into Nanocrystal Superlattices and Glassy Films. J. Am. Chem. Soc. 2006, 128, 3248-3255.

(39) De Benedetti, W. J. I.; Nimmo, M. T.; Rupich, S. M.; Caillard, L. M.; Gartstein, Y. N.; Chabal, Y. J.; Malko, A. V. Efficient Directed Energy Transfer through Size-Gradient Nanocrystal Layers into Silicon Substrates. Adv. Funct. Mater. 2014, 24, 5002-5010.

(40) Yeltik, A.; Guzelturk, B.; Hernandez-Martinez, P. L.; Govorov, A. O.; Demir, H. V. Phonon-Assisted Exciton Transfer into Silicon Using Nanoemitters: The Role of Phonons and Temperature Effects in Förster Resonance Energy Transfer. ACS Nano 2013, 7, 1049210501

(41) Nguyen, H. M.; Seitz, O.; Aureau, D.; Sra, A.; Nijem, N.; Gartstein, Y. N.; Chabal, Y. J.; Malko, A. V. Spectroscopic Evidence for Nonradiative Energy Transfer between Colloidal CdSe/ZnS Nanocrystals and Functionalized Silicon Substrates. Appl. Phys. Lett. 2011, 98, 161904.

(42) Goodfellow, K. M.; Chakraborty, C.; Sowers, K.; Waduge, P.; Wanunu, M.; Krauss, T.; Driscoll, K.; Vamivakas, A. N. DistanceDependent Energy Transfer between CdSe/CdS Quantum Dots and a Two-Dimensional Semiconductor. Appl. Phys. Lett. 2016, 108, No. 021101.

(43) Cabrera, Y.; Rupich, S. M.; Shaw, R.; Anand, B.; de Anda Villa, M.; Rahman, R.; Dangerfield, A.; Gartstein, Y. N.; Malko, A. V.; Chabal, Y. J. Energy Transfer from Colloidal Nanocrystals to Strongly Absorbing Perovskites. Nanoscale 2017, 9, 8695-8702.

(44) Hernández-Martínez, P. L.; Govorov, A. O.; Demir, H. V. Generalized Theory of Förster-Type Nonradiative Energy Transfer in Nanostructures with Mixed Dimensionality. J. Phys. Chem. C 2013, 117, 10203-10212.

(45) Swathi, R. S.; Sebastian, K. L. Long Range Resonance Energy Transfer from a Dye Molecule to Graphene Has (Distance)-4 Dependence. J. Chem. Phys. 2009, 130, 086101.

(46) Prins, F.; Goodman, A. J.; Tisdale, W. A. Reduced Dielectric Screening and Enhanced Energy Transfer in Single- and Few-Layer MoS2. Nano Lett. 2014, 14, 6087-6091.

(47) Hernández-Martínez, P. L.; Govorov, A. O.; Demir, H. V. Förster-Type Nonradiative Energy Transfer for Assemblies of Arrayed Nanostructures: Confinement Dimension vs Stacking Dimension. J. Phys. Chem. C 2014, 118, 4951-4958.

(48) Shafran, E.; Mangum, B. D.; Gerton, J. M. Energy Transfer from an Individual Quantum Dot to a Carbon Nanotube. Nano Lett. 2010, 10, 4049-4054. 